\title{
LA TÉCNICA DEL PRODUCT PLACEMENT EN LAS SERIES DE FICCIÓN GALLEGAS
}

\author{
Sandra Martínez Costa \\ Universidad de A Coruña \\ smartinez@udc.es
}

Material autorizado para su primera publicación en la revista académica Redmarka como artículo

https://doi.org/10.17979/redma.2010.01.05.4715

\section{Resumen}

Este artículo trata sobre la presencia de product placement en las series de ficción en Galicia, para lo cual se ha llevado a cabo el análisis de cuatro de ellas partiendo de la teoría de que las presencia de marcas locales en productos audiovisuales autonómicos pueda resultar más eficaz.

\section{Palabras clave}

Product placement, publicidad, local, series de ficción

\author{
Abstract \\ PRODUCT PLACEMENT TECHNIQUE IN GALICIAN DRAMA
}

This paper deals with the presence of product placement in the fictional series in Galicia. The analysis of these advertising was made in four of the fictional series departing from the theory that local brands in audiovisual products could be more effective.

\section{Keywords}

Product placement, advertising, fictional series 


\section{Introducción}

Pese a que como técnica publicitaria ha sido criticada y venerada casi a partes iguales, el product placement es una de las estrategias below the line más extendidas tanto en el cine como en la ficción televisiva. Su regulación jurídica y aplicación sistemática en grandes países productores como Estados Unidos la han convertido en una técnica imprescindible para recortar gastos y conseguir unos beneficios extraordinarios, que incluyen cada vez más marcas en la ficción audiovisual y los promocionales de sus productos.

En ese sentido, los recientes cambios en la legislación (Ley del Audiovisual, 2009) al respecto de las inserciones de esta modalidad publicitaria favorecerán no solo la regulación en su uso, sino que evitarán también el oscurantismo que muchas productoras y agencias mantenían con respecto de sus actividades en este campo.

Las ventajas de esta modalidad han sido ampliamente reconocidas en el campo publicitario, sobre todo si el formato elegido es el de las series de ficción. Su principal beneficio radica en la imposibilidad de "huida" del espectador ante la marca anunciada a través de prácticas como el zapping, tan frecuente dada la sobreabundancia de cortes publicitarios de larga duración. Pero éste, que podría ser el beneficio más evidente, no es el único. El espectador del producto audiovisual se encuentra perfectamente segmentado y definido, lo que hace muy fácil al anunciante llegar al consumidor y conseguir de él el reconocimiento deseado. Además, las continuas reposiciones y la larga pervivencia de las series favorece su llegada al público durante un largo periodo de tiempo, implicando un alto nivel de asentamiento en el recuerdo del espectador. Si además el programa se vende a otros países, la marca ganará en notoriedad y relevancia.

Por otro lado, la integración con determinados escenarios o con algunos personajes proporciona identidad sin necesidad de contratación de actores, ya 
que la marca se aprovecha de la popularidad e identificación existente entre el público y los protagonistas del producto audiovisual. Además, su inclusión en determinadas escenas cinematográficas aporta credibilidad y veracidad, en especial si su uso queda adecuadamente demostrado en pantalla.

Sin embargo, en el campo de la creación de ficción las ventajas del uso del product placement son más limitadas. Pese a que el emplazamiento de marcas publicitarias caracteriza al personaje y da realismo a la acción, su uso debe ser limitado para no afectar narrativamente a la evolución de la historia y no sobresaturar al espectador. En ocasiones obstaculiza el desarrollo de la ficción y el público no siempre está predispuesto a aceptar la intrusión de la marca en su tiempo de ocio. Como poco está acusada de afectar a los contenidos y al desarrollo del guión y en la mayor parte de las ocasiones, al tipo de planos empleados para determinadas tomas. Al hecho de que esta fórmula publicitaria bordease hasta hace poco la ilegalidad se le suma la falta de estudios sobre su impacto real en la audiencia. En general, la mayor parte de las investigaciones sobre la efectividad de esta tipología publicitaria se centra en los resultados obtenidos del recuerdo o notoriedad en la ubicación del producto, pero muy pocas se adecuan al análisis de su relación con el contenido ficcional. Ello implica que el product placement se aplique de una forma más bien intuitiva y con escasas garantías sobre los resultados previstos, así como sobre su eficacia comunicativa.

A esto se añade el principal inconveniente de que la notoriedad de la marca se halla en función de la propia relevancia del programa así como del interés y la opinión que el público tenga de él. No se trata por lo tanto de variables controlables, ya que el anunciante no puede modificar la predisposición de la audiencia hacia el producto audiovisual. Por esta razón resulta fundamental el adecuado seguimiento de los resultados del programa, así como un estudio previo de su tipología de público, posibilidades de mercado, etc. La guerra por las audiencias y la gran proliferación de series, tanto nacionales como internacionales, hace que los canales muevan la programación a distintas 
franjas horarias con relativa frecuencia o que lleguen incluso a cancelarlas, lo que podría implicar alteraciones en las estrategias del anunciante.

Pese a ello, en términos generales la inclusión de marcas en la ficción audiovisual no suele ir acompañada de otras estrategias publicitarias que hagan más eficaz la inserción, sino que se entiende como una práctica aislada, en muchas ocasiones, del resto de la imagen promocional de la marca. Se da una falta de seguimiento generalizada sobre los resultados, y una escasa experimentación en lo relativo a las propias ubicaciones (excepto prácticas limitadas de escaso éxito, muchas de ellas relacionadas con la interactividad).

En este contexto confuso, la presente investigación pretende centrarse en el estudio de la publicidad insertada en las series de ficción autonómicas (comunidad autónoma gallega). La finalidad es, por un lado, la de estudiar en qué momento se encuentra esta técnica publicitaria en un ámbito de una elevada creación audiovisual, pero en el que los contenidos se encuentran limitados por la barrera idiomática e, incluso en muchas ocasiones, temática. Y por otro lado, la de tratar de reconocer aquellos elementos diferenciadores que definen la inserción de este tipo de productos publicitarios en relación con la identidad cultural de la comunidad autónoma de referencia. De ese estudio se interpretará además el factor diferenciador de la publicidad insertada en las series de ficción autonómicas, y la importancia que ese valor podría tener para la inserción publicitaria de marcas locales. Para ello se observa el posicionamiento de esos productos, su referencia idiomática, la relación con respecto de los personajes y argumentos de la serie, y el refuerzo que pueda suponer para la identidad cultural de la serie / marca de estudio. En ese sentido, el análisis se centra en los siguientes aspectos:

1. La relación entre las audiencias del canal televisivo y la audiencias de la serie en la que se inserta el producto. Asímismo, un tercer elemento de relacion sería entre el target de los productos anunciados y el público de la propia serie. 
2. Por otro lado, se pretende observar si la inclusión de modalidades publicitarias de refuerzo (patrocinio, spots en los bloques de anuncios) podría favorecer la precepción de la marca.

Las hipótesis de partida para el desarrollo de este trabajo se centran, por lo tanto, en lo relativo a las técnicas publicitarias de refuerzo de las marcas.

H1. Las inserciones publicitarias en torno a las series de ficción se hacen con el fin de proporcionar un mayor realismo a la imagen, dándole un carácter identificativo local.

H2. La inserción publicitaria en las series de producción propia en Galicia se ve reforzada por la presencia de anuncios de la misma marca en los cortes previos, posterior y durante la emisión de la serie o con técnicas como la del patrocinio.

Una última hipótesis servirá como punto de partida para la observación del contexto publicitario gallego en lo que a la modalidad de product placement se refiere:

H3. Existen pocas marcas que utilicen el product placement en Galicia como técnica publicitaria continuada, ya que la tendencia es cara a una gran dispersión de pequeños anunciantes que emplean esa modalidad de forma puntual.

Para la observación de estos aspectos se lleva a cabo un estudio descriptivoanalítico a partir de un primer análisis metodológico. El trabajo se centra en el estudio de las audiencias, el product placement de las series, la programación publicitaria que las acompaña y el tipo de emplazamiento que fue llevado a cabo.

Para la realización de un estudio sobre la efectividad del emplazamiento de productos en el entorno de las series de ficción en Galicia se debe llevar a cabo 
una primera fase de análisis, que nos permita identificar las premisas a estudiar de cara al reconocimiento de su efectividad entre el público.

Para ese análisis de contenido seguimos la clasificación hecha por Cristina Olivares y Fernando Del Pino (Olivares, C. \& Del Pino, F., 2006) respecto de la tipología del product placement. Esa clasificación está basada en la relación entre marca o producto y personajes o decorados y se centra en el estudio de los siguientes elementos formales:

1. Contacto físico

1.1. Hiperactivo mención (el personaje interactúa con el producto al mismo tiempo que se refiere a él verbalmente)

1.2. Hiperactivo valoración (el personaje emite una valoración del producto e interactúa con él)

1.3. Verbal mención (hace referencia verbal, pero el producto no tiene porqué estar presente)

1.4. Verbal valoración (valoración expresa, sin presencia física del producto)

1.5. Activo (el personaje manipula el producto)

1.6. Pasivo principal (no hay manipulación del producto, pero éste aparece visiblemente en pantalla)

1.7. Pasivo secundario (el producto no se consume ni se manipula, pero forma parte del atrezzo)

2. Peso narrativo: Podemos hablar de cuatro niveles: muy alto, alto, moderado bajo en función del grado de participación que el producto tenga en la historia.

Para el estudio de la efectividad publicitaria del product placement en el ámbito local se añaden a esta investigación las siguientes variables, referentes a la integración de la marca y el producto en el contexto autonómico gallego:

1. Integración de marcas locales: el nivel de identificación por parte del espectador de las marcas propias de la comunidad autónoma va a depender de 
la propia relevancia que el producto anunciado tenga en el contexto de la serie de ficción.

2. Integración idiomática: el análisis de la integración idiomática se corresponde con el estudio de la lengua en la que esa publicidad se lleva a cabo.

Para Del Pino y Olivares la efectividad del emplazamiento es mayor cuanto más adecuada sea la ubicación del producto en la narración de ficción y, sobre todo, cuanto más regulada esté su exposición y presencia. Si lo que se busca además es llevar a cabo el análisis de la efectividad del mismo a nivel local, se deben tener en cuenta aspectos tales como la dimensión económica de la marca anunciada y el nivel de identificación por parte del espectador. En ocasiones, los productos publicitados a través de esta modalidad en las series de ficción locales son marcas de empresas de la propia ciudad en la que se graba el episodio, con lo que el reconocimiento por parte del target se reduce notablemente. La presencia de esas marcas, aunque favorece a la producción audiovisual desde un punto de vista económico, perjudica la efectividad publicitaria al implicar un mayor nivel de ruido y al mismo tiempo, aumentar los niveles de saturación del espectador que percibe la publicidad aunque no la reconozca.

\section{El sector de la ficción seriada en Galicia}

El éxito de la ficción conseguido en España en los noventa, con series como Médico de Familia o Farmacia de Guardia puso en auge este tipo de contenidos, a la vez rentables económicamente y populares entre una audiencia cada vez más fragmentada. La creación de la Televisión de Galicia (1996) se produjo en los años previos al impulso de este tipo de productos y la fórmula fue adaptada a las condiciones culturales e idiomáticas de la comunidad autónoma. La premisa era la de la cohesión lingüística y social a través de una oferta programática variada con el denominador común de afianzar e integrar la cultura y el idioma gallegos. 
El canal pasó del 69,6\% en 1997 de producción tanto interna como asociada, al $74,4 \%$ en 2000 y al $81,44 \%$ en 2003 , por encima de la media estatal de los restantes canales. En 2003, la ficción ocupaba un 23,7\% de la programación, con apuestas por la producción de series y la emisión de largometrajes gallegos. En ese año fue la televisión de España con mayor porcentaje de series de producción propia, emitiendo más de 500 horas (VV.AA, 2004). Posteriormente aumentó de forma progresiva la emisión de ficción, especialmente de productos de realización externa, hasta llegar a un 33,4\% de la cuota de pantalla en el año 2005 (Fandiño y Dafonte, 2007). En la última década, el volumen de series creadas tanto de forma interna como asociada viene a ser de aproximadamente una docena, algunas de ellas con diez años de pervivencia en antena como en el caso de Pratos Combinados, y otras con gran éxito de audiencia como Mareas Vivas e Padre Casares.

Este volumen de producción de ficción implica que la Televisión de Galicia pasa por ser el motor y la mayor fuente de ingresos para las productoras audiovisuales gallegas, por lo que la conceptualización de las series que éstas realizan se hace pensando que van a ser consumidas por el público potencial del canal autonómico. Las temáticas de los productos de ficción son de contenido localista en todos sus elementos (guión, personajes, escenarios,...) y destinados a la audiencia de una sola emisora, lo que implica una escasa expansión y comercialización de los mismos en mercados nacionales o internacionales.

Destacan las series de contenidos familiares, valores tradicionales y humor "blanco". La mayoría reflejan no solo las peculiaridades diferenciadoras de cada una de las cuatro provincias gallegas, sino que pretenden mostrar las distintas actividades económicas y aspectos sociales de cada una de ellas. Veterinarios, médicos del rural, la industria de la moda, el sector vinícola,... El público se encuentra reflejado, convirtiéndose en audiencia fiel del programa ya que los personajes son marcadamente gallegos y fundamentados en 
estereotipos con el fin de simplificar los argumentos y los gags de los que hacer partícipe al espectador.

Por eso, el interés que este tipo de productos de ficción tiene para la técnica del product placement se centra sobre todo en la eficacia publicitaria que pueda conseguir en un entorno local, en el que los productos anunciados sean marcas reconocidas por su identidad autonómica. La relación entre el localismo de las marcas y el de los productos de ficción hace más fácil la llegada a un público concreto, aunque ello no siempre implica un mejor resultado. Cabe saber, por lo tanto, si esa cercanía con la marca anunciada hace más factible la identificación del espectador con la misma e influye en su percepción y la compra final del producto.

\subsection{Product placement en las series de ficción gallegas.}

En Galicia el uso del product placement es un fenómeno todavía relativamente reciente, que no cuenta con estudios acerca de su empleo y eficacia ni datos sobre ingresos e inversión económica. A día de hoy, su presencia es más bien escasa y desorganizada. El negocio publicitario gallego es ya de por sí reducido y con carencias en cuanto a una estructura que favorezca la compra de espacios de ficción para ser utilizados como soporte de marcas y productos. El volumen de pequeñas agencias es muy elevado, lo que implica dispersión en un mercado que no es excesivamente amplio. Pocas se especializan además en nuevas fórmulas publicitarias en el medio televisivo, con lo que no se cuenta con empresas específicas dedicadas a actividades como el product placement o el patrocinio. Esto, unido al secretismo que se relaciona con este tipo de ocupación, implica que los anunciantes se encuentren ante modelos publicitarios que desconocen, y sobre los que son difíciles de conseguir resultados acerca de su impacto en el consumidor, nivel de recuerdo, etc. De esta forma el sector publicitario es el gran perjudicado, ya que resulta difícil confiar en fórmulas de las que no existen tarifas oficiales o estudios de resultados, descendiendo por lo tanto su uso y efectividad comercial. 
La inserción de product placement en las series de ficción gallegas se hace dependiendo, como norma general, del éxito que éstas tengan, siendo las de mayor audiencia y pervivencia en antena las que incluyen más marcas. En las producciones de varias temporadas de duración es notable el incremento paulatino de anunciantes a lo largo de los años, así como la presencia de patrocinadores. Para favorecer el ejercicio de esta actividad, muchas productoras se esfuerzan ya por incluir en sus series espacios físicos públicos, que narrativamente permitan el encuentro de personajes de distintas esferas sociales y en los que la inserción de marcas resulte natural. La presencia de bares o cafeterías es recurrente, así como las de supermercados, implicando la colocación de un amplio número de marcas variadas, la mayor parte de ellas de tipo autonómico tal y como sucede en series como "Estrella Galicia" y "Agua de Mondariz" en Pratos Combinados y Rías Baixas, respectivamente.

1.2. Análisis del product placement en cuatro series gallegas: Terra de Miranda, Pratos Combinados, Fíos y Rías Baixas

A la hora de analizar la inserción publicitaria en las series de ficción gallegas tendremos en cuenta además no solo los aspectos relatados en los apartados previos, sino también los factores considerados por Lehu (Lehu, J. M., 2007) como necesarios para alcanzar la efectividad buscada:

1. La propia relevancia de la marca: este factor viene condicionado también por el localismo o la internacionalidad del producto anunciado.

2. La identificación de la marca: viene determinada por la ubicación del producto, ya que hay espacios en que éste resulta muy poco reconocible. También va a depender de la notoriedad y experiencia que el público del programa audiovisual tenga al respecto del producto anunciado. En ocasiones el logotipo o nombre de la marca no aparecen claramente identificados, pero el propio envase es suficiente para su reconocimiento. 
3. El presupuesto del producto audiovisual: va a influir en la relevancia de la pieza en la que se inserta la marca anunciada, en el precio del posicionamiento del producto y en la posibilidad de llegar a un segmento de público más o menos amplio.

4. El tipo y el género de producto audiovisual: resulta fundamental a la hora de definir la estrategia publicitaria y también como modo de llegar al público.

5. El crédito del producto audiovisual: es decir, el valor que tienen los elementos que componen ese producto (director, productora, actores, etc.). Se trata de la consideración de su renombre, su fama, imagen o notoriedad entre el público.

6. La importancia del emplazamiento: espacio de pantalla que ocupa el producto y cantidad de veces en que aparece.

7. La localización del emplazamiento: colocación del producto en pantalla la posición que tiene respecto de esta. También si pertenece a escenas de gran intensidad, forma parte del atrezo, etc.

8. Integración en la historia: cuanto mayor es la integración en el argumento o con los personajes, mayor es el impacto provocado en el espectador.

9. Contacto con los actores principales: el contacto entre el actor y el producto resulta ser de una eficacia similar a la de un testimonial, ya que el actor confirma el uso de la marca. A veces, ese mismo actor se emplea para campañas posteriores en el mismo medio u otros.

10. Exclusividad de la marca: el empleo de excesivos productos en una misma imagen audiovisual provoca la saturación en el espectador. Por eso, es fundamental que la marca anunciada esté aislada de otras de la competencia. 
11. Emplazamientos recurrentes: si los emplazamientos seleccionados para la marca están presentes también en otras formas de promoción del producto audiovisual, la notoriedad es mayor. Esto ocurre cuando se utiliza en los teasers, trailers, webs, etc.

12. El tipo de distribución: a mayor internacionalización del producto audiovisual, mayor relevancia para la marca anunciada.

13. Tipos de comunicación que acompañan al producto audiovisual: en ocasiones las campañas de promoción pueden ser incluso compartidas entre el anunciante y la productora.

Estos elementos implican que la técnica del product placement no debe considerarse de forma aislada, sino en relación con la propia relevancia de la marca y con las demás estrategias de comunicación que la acompañan.

Para el estudio del emplazamiento de productos se analizaron cuatro de las series de ficción de la CRTVG en emisión en 2003, durante un periodo de tiempo de una semana. Del análisis de esas cuatro series, se identificaron unas veinticinco marcas, con un arco que va entre las dos de Fíos hasta las once de Pratos Combinados. De la observación de ese contenido publicitario se extraen los siguientes datos:

1. En general el tipo de emplazamento más utilizado es el pasivo, menos eficaz al situarse el producto como un objeto inerte que forma parte de un decorado. Las ubicaciones son casi siempre recurrentes y de baja intensidad, limitando el interés visual. Al no participar de la acción narrativa más que de manera muy pasiva, su valor es escaso y su presencia puede ser molesta si se utiliza en exceso. En ocasiones la posición meramente decorativa de las inserciones hace que se deban generar estrategias de realización audiovisual con la finalidad de remarcar la presencia del producto en pantalla. Los movimientos de cámara juegan el papel de reforzar esa aparición comercial, haciéndole ocupar durante unos instantes un espacio protagonista 
independientemente de su pertinencia. Esta técnica de realización es empleada fundamentalmente en las series Terra de Miranda y Fíos. Por su parte, en Pratos Combinados y Rías Baixas la colocación de las marcas queda más patente gracias al uso de los tiros de cámara. Los encuadres son aprovechados para el desarrollo de escenas de interés dramático en esos espacios, con lo que la presencia del anunciante es obvia, aunque no excesivamente molesta. Esa composición de encuadres destaca sobre todo en el caso de los decorados de los bares y lugares públicos presentes en las cuatro series analizadas.

2. De las marcas anunciadas en las series, casi la mitad (10) son de carácter local o autonómico. La mayor parte de los emplazamientos son de marcas de productos con un mercado nacional (8) e internacional (5). Sin embargo, la frecuencia de de inserciones es mayor en el caso de las locales o autonómicas, tal y como sucede con algunas como "Estrella Galicia" (15 por episodio), "Santiveri" (10 por episodio), "Sargadelos" y "Auga de Mondariz" en Rías Baixas o "Maselga" en Fíos, mientras que las nacionales e internacionales tienen una presencia menos significativa. Esta abundancia de impactos de marcas locales hace que su visibilidad sea aún más notable.

3. La relación directa entre la temática de las series y el contenido publicitario coincide en muy escasas ocasiones. Aunque cabría esperar que en series como Fíos, con un contenido relacionado con el sector de la moda, los anunciantes fuesen marcas textiles, o en Rías Baixas alguno de los vinos gallegos (albariño, fundamentalmente dada la zona geográfica en la que se ubica la serie) lo cierto es que no ocurre así. Destaca por el contrario la presencia de marcas como "Biona", empresa de productos destinados al campo gallego, en una serie específica de la temática del rural y los veterinarios: Terra de Miranda. En este caso, la ubicación resulta más efectiva al aplicarse tan solo al espacio de la agropecuaria. La relación entre el tema central de la serie y el producto anunciado resulta en este caso pertinente y, por lo tanto, más efectiva. 
4. La relación entre el personaje y la marca anunciada es escasa, lo que implica una reducida presencia de contacto físico activo, verbal o hiperactivo. Solo en muy pocos casos el producto se integra como un elemento definidor del carácter de alguno de los personajes, pese a la notable relación personajemarca que se establece entre Antón Santos y "Estrella Galicia" en la serie Pratos Combinados, por ejemplo. En su papel de vividor, mujeriego y tramposo Antón destaca también por su gusto por las nécoras y las cervezas de su hermana Balbina. Antón no solo bebe a todas horas la marca "Estrella Galicia" sino que ésta está presente en su piso gracias a un cartel publicitario de "1906" (producto de la misma compañía) así como a numerosos packs, latas y botellas desperdigados por cada rincón. La ubicación en el espacio íntimo del personaje implica que éste y la marca quedan unidos y claramente relacionados. Mientras, "Estrella Galicia" refuerza su carácter gallego, idea fuerza de sus campañas publicitarias en los últimos años, al marcar la relación con el personaje de Antonio Durán "Morris", actor reconocido en el ámbito cultural de la comunidad autónoma.

5. Solo en muy raros casos existe una relación directa entre el público objetivo publicitario y la serie anunciada. El espectador de las series analizadas está en un segmento de edad de más de 45 años y de ámbito rural, pese a lo cual se dan abundantes casos en los que el target de la marca anunciada no se corresponde con ese perfil. "Estrella Galicia" cuenta, por ejemplo, con un consumidor mayoritariamente de edad joven, pese a lo cual ha utilizado este tipo de series de ficción para generar imagen de marca.

6. A pesar de la presencia de anunciantes locales no hay en las series de ficción analizadas ninguna publicidad hecha exclusivamente en gallego, excepto si se consideran como tal las escasas menciones verbales de carácter turístico sobre algunas de las ciudades en las que se han grabado las series. Pese a que hay carteles comerciales en los decorados, los mensajes están siempre en castellano. 


\section{Conclusiones}

En general, las líneas argumentales de carácter local y autonómico favorecen la presencia de marcas gallegas, que son empleadas para reforzar el carácter de determinados personajes produciéndose una sinergia de creación de identidad cultural. En muchos casos se emplean marcas o comercios propios del lugar en el que se lleva a cabo el rodaje del programa, favoreciendo la conexión con un público que busca el acercamiento a su propia cultura y a la realidad cotidiana de su mundo y amortizando en parte los gastos de producción. La identidad que muestran marcas tan populares entre los gallegos como "Larsa", "Sargadelos" o "Estrella Galicia" aparece reforzada al entrar en contacto con la ficción televisiva gallega, en mundos en los que las bodegas de albariño o las agropecuarias forman parte de la vida del espectador.

Las inserciones se realizan en general con el fin de proporcionar un mayor realismo a la imagen, así como de remarcar la identidad gallega de determinados personajes o situaciones. El hecho de que muchas de las marcas anunciadas sean de productos locales, implica que éstas proporcionan un tono identificativo a la narración aunque no siempre exista una relación directa entre el contenido temático y la publicidad.

Sin embargo, es todavía difícil de definir la eficacia que su uso tiene sobre el espectador. La identificación de la marca no siempre se lleva a cabo, ya que en ocasiones las inserciones publicitarias son de empresas no reconocidas más allá de la ciudad en la que llevan a cabo su actividad económica principal. No generan por lo tanto la identificación emocional que debieran en su público, pero en cambio sí son percibidas como publicidad intrusiva por parte del espectador.

La inexistencia de una estructura organizada de agencias de publicidad dedicadas al product placement en Galicia implica que la tarea de selección de emplazamientos se hace desde la propia productora que realiza la serie o desde la empresa anunciante, que negocia en muchos casos la inserción 
publicitaria. La finalidad es la de encontrar el mayor espacio posible en pantalla o conseguir el primer plano del producto, en lugar de emplear estrategias comunicativas más efectivas. Por esta razón, entre otras, se incluyen marcas locales no reconocibles más allá de un pequeño porcentaje de la audiencia, sin tener en cuenta el efecto negativo de dicha inserción sobre las otras marcas presentes en la serie, o sobre el propio espectador. Además el uso de este tipo de emplazamientos limita la eficacia publicitaria de los mismos a un contexto geográfico y cultural muy reducido, perjudicando a aquellas marcas con un mercado más amplio que el autonómico que podrían aprovechar el beneficio que supone la venta del producto audiovisual a nivel nacional o internacional.

Sin embargo, es cierto que la percepción del espectador en aquellos casos en los que el emplazamiento es adecuado podría ser más emotiva si identifica la marca con su raíces culturales y geográficas más cercanas. Por ello, en determinados productos audiovisuales planteados para una difusión reducida y un consumo local, se gana en efectividad publicitaria con la inclusión de pequeñas marcas que generen identidad cultural. Sin embargo, para que este emplazamiento resulte efectivo es necesario algo más que una simple ubicación. Un correcto seguimiento sobre los resultados del emplazamiento, así como una adecuada campaña de comunicación global y de publicidad en los medios de comunicación podría facilitar el reconocimiento del público de cara a la marca, resultando más eficaz.

\section{Bibliografía}

DEL PINO, C \& OLIVARES, F. (2006). Brand placement: integración de marcas en la ficción audiovisual. Evolución, casos, estrategias y tendencias. Barcelona, Gedisa.

FANDIÑO ALONSO, X. \& DAFONTE GÓMEZ, A. (2007). "A televisión en Galicia: actualidade e previsión de futuro". A comunicación en Galicia 2007 (Coord. X. L. García). Santiago de Compostela, Consello da Cultura Galega, p.95. 
GALICIAN, M. L. (2004) Handbook of product placement in the mass media. New strategies in marketing theory, practice, trends and ethics. Haworth Press: Estados Unidos.

LEHU, J. M. (2007) Branded Entertainment. Product Placement \& Brand Strategy in the Entertainment Business. MPG Books, Cornwall.

MENDIZ NOGUERO, A. (2000) Nuevas Formas Publicitarias: Patrocinio, Product Placement, Publicidad e Internet. Universidade de Málaga: Málaga.

ORTEGA MARTÍNEZ, E. (1992). La publicidad en televisión. Madrid, Mundiprensa.

VV. AA. (2004). A Comunicación en Galicia 2004. Santiago de Compostela, Consello da Cultura Galega, pp. 50-51.

\section{Para citar este artículo:}

Martínez Costa, Sandra (17-12-2010). LA TÉCNICA DEL PRODUCT PLACEMENT EN LAS SERIES DE FICCIÓN GALLEGAS.

REDMARKA - CIECID - Unidad de Investigaci $\tilde{A}^{3} n$ en Marketing Aplicado-Universidad de A CoruÃ $\pm a$ Año II, Número 5, V1, pp.77-93PRODU

ISSN $1852-2300$

URL del Documento : cienciared.com.ar/ra/doc.php?n=1415

URL de la Revista : cienciared.com.ar/ra/revista.php?wid=39 